\title{
Habitual fish consumption and risk of incident stroke: the European Prospective Investigation into Cancer (EPIC)-Norfolk prospective population study
}

\author{
Phyo K Myint ${ }^{1, *} \dagger$, Ailsa A Welch ${ }^{1}$, Sheila A Bingham², Robert N Luben ${ }^{1}$, \\ Nicholas J Wareham ${ }^{3}$, Nicholas E Day ${ }^{1}$ and Kay-Tee Khaw ${ }^{1}$ \\ ${ }^{1}$ Department of Public Health and Primary Care, University of Cambridge, UK: ${ }^{2}$ MRC Dunn Human Nutrition Unit, \\ Cambridge, UK: ${ }^{3}$ MRC Epidemiology Unit, Elsie Widdowson Laboratories, Cambridge, UK
}

Submitted 22 August 2005: Accepted 10 January 2006

\begin{abstract}
Objectives: To examine the association between fish consumption and stroke risk. Design: Prospective population cohort study.

Setting: Norfolk, UK cohort of the European Prospective Investigation into Cancer (EPIC-Norfolk).

Subjects: Subjects were 24312 men and women aged 40-79 years who had no previous history of stroke at baseline.

Methods: Fish consumption was assessed using a food-frequency questionnaire at baseline in 1993-1997 and stroke incidence ascertained to 2004.

Results: A total of 421 incident strokes were identified (mean follow-up $=8.5$ years, total person-years $=209238$ ). There were no significant relationships between total fish, shellfish or fish roe consumption and risk of stroke in men and women after adjusting for age, systolic blood pressure, body mass index, smoking, cholesterol, diabetes, physical activity, alcohol consumption, fish oil supplement use and total energy intake using Cox regression analyses. Oily fish consumption was significantly lower in women who subsequently had a stroke (odds ratio (OR) for consumers vs. non-consumers $=0.69,95 \%$ confidence interval (CI) $0.51-0.94, P=0.02)$. The trend in men was similar but not significant (OR for consumers vs. non-consumers $=0.88$, 95\% CI $0.65-1.19, P=0.41$ ).

Conclusions: There was no consistent relationship between fish consumption and stroke in this British population. Inconsistencies in the observed health effects of fish consumption in different populations may reflect different patterns and type of fish consumed and preparation methods.
\end{abstract}

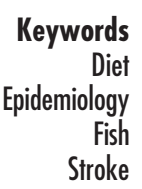

Stroke is a preventable condition. There is increasing interest in lifestyle, in particular dietary factors, which may reduce stroke risk and which may or may not be mediated through known risk factors such as raised blood pressure.

Observational and interventional studies suggest that a high intake of $n-3$ fatty acids, which are mainly derived from fish and seafood, may be cardioprotective, although the evidence is not entirely consistent ${ }^{1,2}$. The protective effect of fish consumption on risk of stroke remains more equivocal $^{3-5}$. A recent meta-analysis of cohort studies by He et al. concluded that there appears to be a beneficial relationship between fish consumption and ischaemic stroke. However, due to the nature and limited number of the studies included, the authors acknowledged the need for further clarification ${ }^{6}$.

†Correspondence address: Room 311, Strangeways Research Laboratory, Wort's Causeway, Cambridge CB1 8RN, UK.
Some of the inconsistencies may reflect not just the amount of fish consumed but also other factors - such as type, composition and preparation methods, etc. - that may vary in different populations. In the present study, we prospectively examined habitual fish consumption and incident stroke (fatal and non-fatal) among participants in the British (Norfolk) arm of the European Prospective Investigation into Cancer (EPIC-Norfolk).

\section{Materials and methods}

\section{Study population}

The study population is free-living men and women aged 40-79 years at the time of recruitment (1993-1997) who were identified from general practice registers and invited to participate in EPIC-Norfolk ${ }^{7}$. The Norwich Local Research Ethics Committee approved the study. 


\section{Measurements}

At the baseline assessment in 1993-1997, measures were made by trained staff according to standardised protocols ${ }^{8}$. Blood pressure was measured using an Accutorr sphygmomanometer after the participant had been seated resting for $3 \mathrm{~min}$. The mean of two blood pressure readings was used in analysis. Body mass index (BMI) was calculated as weight in kilograms divided by the square of height in metres $\left(\mathrm{kg} \mathrm{m}^{-2}\right)$. Non-fasting blood samples were taken. Serum levels of total cholesterol, high-density lipoprotein cholesterol and triglycerides were measured on fresh samples with an RA 1000 analyser (Bayer Diagnostics, Basingstoke, UK), and low-density lipoprotein cholesterol levels were calculated with the Friedewald formula?

Participants were asked 'Have you ever smoked as much as one cigarette a day for as long as a year?' and 'Do you smoke cigarettes now?' in the baseline questionnaire. From these responses, people were classified as current smokers, ex-smokers or those who had never smoked. On the health questionnaire, the participants were asked 'Has a doctor ever told you that you have any of the following?' followed by a list of conditions including heart attack, stroke, cancer and diabetes to obtain baseline prevalence of illnesses.

Alcohol consumption was derived from a foodfrequency questionnaire (FFQ) collected at baseline. For the 'drinks' category, responses ranging from never to more than six times per day were given for four types of alcoholic drink. An in-house computer program, CAFE, was developed for data entry and analysis ${ }^{10}$.

In the FFQ, participants were also asked to report intake of food supplements including fish oils. If the response was 'yes', then the participants were asked to provide the full name, brand, strength and dose (number of pills, capsules or teaspoons consumed) and also to choose a response ranging from never or less than once a month to more than six times per day. For fish oils, the responses were dichotomised as consumers and non-consumers. Estimated daily total energy intake (kcal) was calculated by the CAFE program using food tables and portion weighing.

A four-level physical activity index (level I = inactive, II = moderately inactive, III = moderately active, $\mathrm{IV}=$ active) was derived from the EPIC short physical activity questionnaire designed to assess combined work and leisure activity. The validity and repeatability of this scoring system have been detailed elsewhere ${ }^{11}$.

\section{Predictor variable}

Under the 'meat and fish' section of the FFQ, participants were asked their average fish consumption in the previous year. We asked the participants 'Please estimate your average food use as best you can, and please answer every question - do not leave any lines blank. Please put a tick $(\sqrt{ })$ on every line'. They were asked to choose responses ranging from never to more than six times per day of a medium portion for four types of fish: white fish including cod, haddock, plaice, sole and halibut - either fresh or frozen, fried fish in batter (e.g. fish and chips), fish fingers and fish cakes; oily fish such as mackerel, kippers, tuna, salmon, sardines and herring - either fresh or canned; shellfish such as crab, prawns, mussels; and fish roe and taramasalata. Total fish consumption was derived from the combination of any fish consumed. Fish consumption in portions per week was estimated for total fish, white fish, oily fish, shellfish and fish roe (fish roe and taramasalata).

\section{Outcome measures}

All individuals have been flagged for death certification at the UK Office of National Statistics with vital status ascertained for the whole cohort. A hospital record linkage system (ENCORE) also enabled identification of causespecific hospital episodes for all participants. Incident stroke cases were identified by combining mortality and hospital episodes using the Tenth Revision of the International Classification of Diseases (ICD-10), codes I60-I69. We present results for the cohort followed up to 2004 , an average follow-up of about 8.5 years.

\section{Statistical analyses}

Statistical analyses were performed using SPSS for Windows version 12.0.1 (SPSS Inc., Chicago, IL, USA). We excluded participants with self-reported stroke at baseline. Participants with missing values for covariates used in different models were excluded in individual regression analyses.

We examined the sex-specific association between fish consumption and the risk of stroke by performing separate analyses for total fish, white fish, oily fish, shellfish and fish roe. We used the Cox proportional hazards model ${ }^{12}$ to determine the independent contributions of fish consumption and other covariates. First we adjusted for increasing age by 5 years (continuous), increasing systolic blood pressure by $10 \mathrm{mmHg}$ (continuous), BMI (continuous), cigarette smoking status (current smoker, ex-smoker, never-smoked), cholesterol level (continuous) and diabetes (yes/no). In the second model, we additionally adjusted for fish oil supplement (yes/no), physical activity level (I-IV), average alcohol consumption in $\mathrm{g} \mathrm{day}^{-1}$ (continuous) and total energy intake in $\mathrm{kcalday}^{-1}$ (continuous). The purpose of this stepped approach was twofold: (1) to examine the relationship of fish consumption to stroke risk independently of classical risk factors through which fish and fish oils may influence health; and (2) to examine the possible confounding associated with other lifestyle behaviours.

\section{Results}

There were 24312 participants (10972 men and 13340 women) included in the study after excluding 455 participants with prevalent stroke. There were 421 incident stroke cases up to the end of July 2004 excluding 
self-reported incident cases (total person-years $=209238$, mean follow-up of 8.5 years).

Table 1 shows the distribution of sample characteristics at baseline for incident stroke cases ascertained by record linkage $(n=421$, fatal stroke $=141)$ and the rest of the cohort. In both men and women, those who subsequently had a stroke were older, had higher mean systolic blood pressure, cholesterol, a higher proportion of people with diabetes, and were physically inactive at baseline. Total fish and white fish consumption were significantly higher among men who developed incident stroke, although the absolute magnitude of differences was small. The 10th and 90th percentile values for total fish consumption (portions per week) in those who did not develop stroke were 0.5 and 4.0 and in those who developed incident stroke were 0.5 and 4.5 , respectively. In women, the corresponding values for both those who did not develop stroke and who developed stroke were 0.5 and 4.5 . Oily fish consumption was significantly lower in women who subsequently developed a stroke.

Table 2 shows the sex-specific crude event rate and adjusted relative risks for stroke by three categories of total fish consumption $(<1,1-2$ and $>2$ portions per week), firstly adjusting for age, secondly adjusting for age, systolic blood pressure, BMI, smoking, cholesterol and diabetes and then additionally adjusting for fish oil supplement use, physical activity, alcohol consumption and total energy intake. There was no significant relationship between fish consumption and stroke. Similar associations were observed for white fish consumption (results not shown).

Table 3 shows the sex-specific relative risk of incident stroke between those who consumed oily fish, shellfish and fish roe and those who did not, using similar models to those in Table 2. Oily fish consumption in women

Table 1 Distribution of characteristics of incident stroke cases and cohort in 24312 men and women aged $40-79$ years without history of stroke at baseline in EPIC-Norfolk, 1993-2004

\begin{tabular}{|c|c|c|c|}
\hline & $\begin{array}{l}\text { No incident } \\
\text { stroke }\end{array}$ & $\begin{array}{l}\text { Incident } \\
\text { stroke }\end{array}$ & $P$-value \\
\hline Men & $n=10755$ & $n=217$ & \\
\hline Age (years) & $58.9(9.3)$ & $66.5(7.6)$ & $<0.0001$ \\
\hline BMI $\left(\mathrm{kg} \mathrm{m}^{-2}\right)$ & $26.5(3.3)$ & $26.7(3.2)$ & 0.32 \\
\hline $\mathrm{SBP}(\mathrm{mmHg})$ & 137 (17.5) & $150(20.0)$ & $<0.0001$ \\
\hline Cholesterol (mmoll $\left.{ }^{-1}\right)$ & $6.0(1.1)$ & $6.2(1.2)$ & 0.04 \\
\hline Smokers, current & $1286(12.0 \%)$ & $33(15.3 \%)$ & $0.03^{*}$ \\
\hline History of diabetes & $313(2.9 \%)$ & $16(7.4 \%)$ & $<0.0001$ \\
\hline Cod liver oil supplement (yes) & 2894 (26.9\%) & $53(24.4 \%)$ & 0.41 \\
\hline Physical activity & & & $<0.0001^{*}$ \\
\hline Inactive & $3053(28.4 \%)$ & $100(46.1 \%)$ & \\
\hline Moderately inactive & $2721(25.3 \%)$ & $56(25.8 \%)$ & \\
\hline Moderately active & $2521(23.4 \%)$ & $27(12.4 \%)$ & \\
\hline Active & $2460(22.9 \%)$ & $34(15.7 \%)$ & \\
\hline Alcohol $\left(\right.$ g day $\left.^{-1}\right)$ & $12.3(16.0)$ & $12.0(17.4)$ & 0.76 \\
\hline Energy $\left(\mathrm{KJ} \mathrm{day}^{-1}\right)$ & $9180(2624)$ & $9138(2763)$ & 0.82 \\
\hline Total fish (frequency week ${ }^{-1}$ ) & $2.2(1.6)$ & $2.4(1.8)$ & 0.05 \\
\hline White fish (frequency week ${ }^{-1}$ ) & $1.3(1.0)$ & $1.4(1.1)$ & 0.04 \\
\hline Shellfish (frequency week ${ }^{-1}$ ) & $0.2(0.4)$ & $0.2(0.5)$ & 0.46 \\
\hline Oily fish (frequency week ${ }^{-1}$ ) & $0.7(0.9)$ & $0.7(1.0)$ & 0.49 \\
\hline Fish roe (frequency week ${ }^{-1}$ ) & $0.04(0.2)$ & $0.05(0.4)$ & 0.24 \\
\hline Women & $n=13136$ & $n=204$ & \\
\hline Age (years) & $58.2(9.2)$ & $67.0(7.9)$ & $<0.0001$ \\
\hline BMI $\left(\mathrm{kg} \mathrm{m}^{-2}\right)$ & $26.2(4.3)$ & $26.8(4.6)$ & 0.051 \\
\hline $\mathrm{SBP}(\mathrm{mmHg})$ & $134(19.0)$ & $146(20.2)$ & $<0.0001$ \\
\hline Cholesterol (mmoll $\left.{ }^{-1}\right)$ & $6.3(1.2)$ & $6.6(1.3)$ & 0.003 \\
\hline Smokers, current & $1454(11.2 \%)$ & $32(15.8 \%)$ & $0.09^{*}$ \\
\hline History of diabetes & $190(1.4 \%)$ & $12(5.9 \%)$ & $<0.0001$ \\
\hline Cod liver oil supplement (yes) & $4377(33.3 \%)$ & $66(32.4 \%)$ & 0.77 \\
\hline Physical activity & & & $<0.0001^{*}$ \\
\hline Inactive & 3657 (27.8\%) & 99 (48.5\%) & \\
\hline Moderately inactive & $4308(32.8 \%)$ & $58(28.4 \%)$ & \\
\hline Moderately active & $3019(23.0 \%)$ & $31(15.2 \%)$ & \\
\hline Active & 2152 (16.4\%) & $16(7.8 \%)$ & \\
\hline Alcohol $\left(\right.$ g day $\left.^{-1}\right)$ & $5.6(8.4)$ & $4.1(7.6)$ & 0.009 \\
\hline Energy $\left(\mathrm{KJ} \mathrm{day}^{-1}\right)$ & $8082(2287)$ & 8109 (2466) & 0.87 \\
\hline Total fish (frequency week ${ }^{-1}$ ) & $2.3(1.7)$ & $2.2(1.5)$ & 0.40 \\
\hline White fish (frequency week ${ }^{-1}$ ) & $1.3(1.0)$ & $1.4(1.0)$ & 0.19 \\
\hline Shellfish (frequency week ${ }^{-1}$ ) & $0.2(0.5)$ & $0.2(0.4)$ & 0.33 \\
\hline Oily fish (frequency week ${ }^{-1}$ ) & $0.8(0.9)$ & $0.6(0.8)$ & 0.011 \\
\hline Fish roe (frequency week ${ }^{-1}$ ) & $0.04(0.2)$ & $0.05(0.3)$ & 0.85 \\
\hline
\end{tabular}

EPIC - European Prospective Investigation into Cancer and Nutrition; BMI - body mass index; SBP - systolic blood pressure. Values are expressed as mean (standard deviation) unless specified otherwise.

${ }^{*}$ Overall $P$-value. 
showed a significant inverse relationship with risk of stroke (relative risk $(\mathrm{RR})=0.69,95 \%$ confidence interval (CI) $0.51-0.94, P=0.02$ ).

The caveat is that while for total fish there was no consistent relationship overall, for oily fish there appeared to be an inverse association in both men and women, with a combined-sex RR of 0.78 (95\% CI 0.63-0.97, $P=0.024$ ) after adjustments. This needs to be confirmed in future studies.

\section{Discussion}

In this prospective study of a British population with average follow-up of 8.5 years, we found no evidence that higher total fish consumption is associated with reduced risk of subsequent stroke. However, there appeared to be an inverse association between oily fish consumption and the relative risk of stroke in women.

Although ecological or cross-sectional and case-control studies have reported an inverse association between consumption of fish and fish oils and stroke risk, results from prospective studies have been less consistent ${ }^{13}$. Moreover, despite the pooled data reported by He et al. from nine cohorts suggesting a beneficial effect of fish consumption two to four times per week compared with less than once per month, individual cohort analyses showed varying results ${ }^{6}$.

Whether differences in the results from cohort studies may relate to differences in the type of fish consumption in different populations or differences in the overall characteristics of the sample populations and/or type of stroke is unclear. For example, Keli et al. performed their study in a population with relatively high oily fish consumption $^{14}$. Moreover, data reported from the USA showed differences in associations between sexes: while the Nurses' Health Study ${ }^{15}$ suggested a benefit of consumption of fish and omega-3 polyunsaturated fatty acids on risk reduction of thrombotic infarction, Morris et al. reported that there was no convincing evidence to support moderate fish consumption and lower stroke risk in men in the Physicians' Health Study ${ }^{16}$. Similarly, conflicting results have been shown between sexes: women but not men who consumed fish more than once per week had lower risk than those who had never consumed fish in the National Health and Nutrition Examination Survey I study ${ }^{17}$.

The gender difference observed in the current study and others may be explained by differences in genetic and hormonal make-up between men and women. Although we adjusted for several variables including smoking, physical activity and total energy intake, we could not exclude residual confounding. We did not adjust for psychosocial factors which differ between sexes, e.g. hostility, aggression, etc., which may play a role in cardiovascular health. It is also possible that men and women report stroke differentially and there were more women than men with milder strokes in this cohort, although this seems less likely.

Most of the studies did not present data for specific type of fish consumed. Mozaffarian et al. reported that consumption of tuna or boiled or baked fish was associated with lower risk of ischaemic stroke, while intake of fried fish or fish sandwiches was associated with a higher risk, suggesting that how fish is prepared could influence the relationship ${ }^{18}$.

The numbers of stroke events in this study and hence the statistical power are comparable to those in other studies that reported a significant relationship between fish intake and stroke. In this cohort, white fish is the predominant contributor to total fish consumption. It is possible that any cardiovascular protective effect of fish may be due to $n-3$ fatty acids. White fish is not rich in $n-3$ fatty acids.

Table 2 Overall numbers of events, rates, crude and adjusted relative risk for three categories of total fish consumption using ICD-10 coding

\begin{tabular}{|c|c|c|c|c|}
\hline & \multicolumn{3}{|c|}{ Total fish consumption (portions week ${ }^{-1}$ ) } & \multirow[b]{2}{*}{$P$-value } \\
\hline & $<1$ & $1-2$ & $>2$ & \\
\hline \multicolumn{5}{|l|}{ Men } \\
\hline Number & 3049 & 3814 & 4109 & \\
\hline Rate/100 (events) & $1.7(53)$ & $2.0(75)$ & $2.2(89)$ & 0.44 \\
\hline Relative risk* & 1.00 & $1.07(0.75-1.52) \S$ & $1.23(0.88-1.73)$ & 0.45 \\
\hline Relative risk $†$ & 1.00 & $1.12(0.77-1.64)$ & $1.33(0.93-1.92)$ & 0.27 \\
\hline Relative riskł & 1.00 & $1.12(0.76-1.63)$ & $1.34(0.93-2.93)$ & 0.26 \\
\hline \multicolumn{5}{|l|}{ Women } \\
\hline Number & 3282 & 4562 & 5496 & \\
\hline Rate/100 (events) & $1.9(63)$ & $1.3(60)$ & $1.5(81)$ & 0.09 \\
\hline Relative risk* & 1.00 & $0.66(0.46-0.93)$ & $0.76(0.55-1.06)$ & 0.06 \\
\hline Relative risk $†$ & 1.00 & $0.72(0.50-1.05)$ & $0.81(0.57-1.16)$ & 0.22 \\
\hline Relative riskł & 1.00 & $0.74(0.51-1.08)$ & $0.86(0.60-1.24)$ & 0.29 \\
\hline
\end{tabular}

ICD-10 - Tenth Revision of the International Classification of Diseases.

*Adjusted for increasing age.

† Adjusted for increasing age, increasing systolic blood pressure, body mass index, smoking, cholesterol and diabetes. $\ddagger$ Additionally adjusted for fish oil supplement use, physical activity, alcohol consumption and total energy intake.

$\S 95 \%$ confidence interval. 
Table 3 Overall numbers of events, rates, crude and adjusted relative risk for oily fish, shellfish and fish roe consumption using ICD-10 coding

\begin{tabular}{|c|c|c|c|}
\hline & \multicolumn{2}{|c|}{ Fish consumption } & \multirow[b]{2}{*}{$P$-value } \\
\hline & No & Yes & \\
\hline \multicolumn{4}{|l|}{ Oily fish } \\
\hline Number & 3506 & 7466 & \\
\hline Rate/100 (events) & $2.2(78)$ & $1.9(139)$ & 0.20 \\
\hline Relative risk ${ }^{*}$ & 1.00 & $0.87(0.65-1.16) \ddagger$ & 0.34 \\
\hline $\begin{array}{l}\text { Relative risk } † \\
\text { Women }\end{array}$ & 1.00 & $0.88(0.65-1.19)$ & 0.41 \\
\hline Number & 3239 & 10101 & \\
\hline Rate/100 (events) & $2.3(73)$ & $1.3(131)$ & $<0.0001$ \\
\hline Relative risk ${ }^{*}$ & 1.00 & $0.67(0.49-0.91)$ & 0.009 \\
\hline Relative risk† & 1.00 & $0.69(0.51-0.94)$ & 0.019 \\
\hline \multicolumn{4}{|l|}{$\begin{array}{l}\text { Shellfish } \\
\text { Men }\end{array}$} \\
\hline Number & 7922 & 3050 & \\
\hline Rate/100 (events) & $2.1(164)$ & $1.7(53)$ & 0.26 \\
\hline Relative risk* & 1.00 & $1.02(0.73-1.41)$ & 0.93 \\
\hline $\begin{array}{l}\text { Relative risk } \dagger \\
\text { Women }\end{array}$ & 1.00 & $0.99(0.71-1.37)$ & 0.93 \\
\hline Number & 9205 & 4135 & \\
\hline Rate/100 (events) & $1.7(154)$ & $1.2(50)$ & 0.043 \\
\hline Relative risk* & 1.00 & $0.95(0.68-1.33)$ & 0.75 \\
\hline Relative risk† & 1.00 & $1.08(0.71-1.42)$ & 0.97 \\
\hline \multicolumn{4}{|l|}{$\begin{array}{l}\text { Fish roe } \\
\text { Men }\end{array}$} \\
\hline Number & 10294 & 678 & \\
\hline Rate/100 (events) & $2.0(203)$ & $2.1(14)$ & 0.87 \\
\hline Relative risk ${ }^{*}$ & 1.00 & $1.12(0.62-2.01)$ & 0.71 \\
\hline $\begin{array}{l}\text { Relative risk } † \\
\text { Women }\end{array}$ & 1.00 & $1.13(0.63-2.03)$ & 0.69 \\
\hline Number & 12447 & 893 & \\
\hline Rate/100 (events) & $1.5(192)$ & $1.3(12)$ & 0.64 \\
\hline Relative risk* & 1.00 & $1.23(0.68-2.20)$ & 0.50 \\
\hline Relative risk $\dagger$ & 1.00 & $1.34(0.74-2.41)$ & 0.34 \\
\hline
\end{tabular}

ICD-10 - Tenth Revision of the International Classification of Diseases. ${ }^{*}$ Adjusted for increasing age, increasing systolic blood pressure, body mass index, smoking, cholesterol and diabetes.

† Additionally adjusted for fish oil supplement use, physical activity, alcohol consumption and total energy intake.

$\ddagger 95 \%$ confidence interval.

Additionally, the method of fish preparation in the UK where fish is usually eaten after heavy processing, e.g. battered and fried as in fish and chips or as fish fingers, etc. - may also not result in overall cardiovascular benefit. In a comparative calibration study of the variability of fish consumption within European countries in the EPIC study, Welch et $\mathrm{al}^{19}{ }^{19}$ reported that approximately 30\% of the fish consumed in the UK is cod, $6 \%$ herring and $14 \%$ salmon, in comparison with the Netherlands, where the highest categories of fish consumptions are oily fish such as herring (22\%) and salmon (19\%).

It is possible that a period longer than 10 years' exposure is required for the observed beneficial effect of fish consumption on risk of stroke. The studies which showed an inverse association between fish intake and stroke risk generally had follow-up for at least 12 years $3,14,15,17$. However, Orencia et al. did not find the inverse association in a 30-year follow-up study ${ }^{20}$. Additionally, it does not seem biologically plausible that a long period of exposure is required as the mechanism of action of fish or fish oil is thought to be through antithrombotic, antiinflammatory or anti-arrhythmic activity ${ }^{21,22}$ and the intervention trials certainly suggest short-term effects ${ }^{1,23}$.

There are limitations in our study. Because participants had to be willing to provide detailed information and participate in a long-term follow-up study, the response rate was only $40-45 \%$ for the baseline and follow-up. Nevertheless, the characteristics of this population are comparable to national samples ${ }^{7}$. Fish consumption was derived from a single FFQ at baseline and we have no information on change in fish intake over the study period. Nevertheless, fish consumption is relatively stable and shows less inter-individual variability than most other dietary factors.

We identified stroke cases by death certificates and a hospital record linkage system using ICD-10 codes I60I69. This may not capture less severe strokes and is likely to result in underestimation of incident cases. It is unlikely that fish consumption would relate differentially to milder strokes unless we believed that there is wide variation in the mechanism of action of fish and fish oils in different individuals. Moreover, the stroke cases identified in these analyses are likely to be those with the most clinical impact. Misclassification of strokes is likely to attenuate any relationships. It could be argued that our results are obscured by differences in the relationship between fish consumption and stroke subtypes, particularly ischaemic and haemorrhagic strokes, as a beneficial effect of fish consumption has been more closely linked to ischaemic stroke than to haemorrhagic stroke ${ }^{13,18}$. However, the latter represents only $10-15 \%$ in this Caucasian population and therefore, while this may attenuate any associations, this should not influence the direction of the relationship.

Only 210 out of 421 cases ascertained by record linkage had radiological confirmation and stroke subtype could be accurately classified only in 160 (ischaemic stroke $=121$, haemorrhagic $=39$ ), although the remaining 50 cases could be presumed as ischaemic subtype. With these low numbers, we did not have the power to examine different subtypes of stroke.

In this cohort, there appeared to be no consistent relationship between fish consumption and stroke risk despite the average total fish consumption (portions per week) being comparable to the level of fish consumption associated with cardiovascular benefit ${ }^{1}$. However, the caveat is that in the former study (the Diet and Reinfarction Trial) reduction in mortality in men who had myocardial infarction was associated with a modest intake of fatty fish (two or three portions per week) not with any type of fish ${ }^{1}$. Although total fish consumption in our cohort is comparable to that in other European countries, oily fish consumption is not high (Table 1) and this may partly explain the inconsistent association observed ${ }^{19}$. 
It is possible that any protective effect of fish consumption on stroke risk may not be generalisable. High fish consumption may be an indicator of other dietary patterns or other behaviours that may be protective for stroke, such as lower fat or higher fruit and vegetable intake, and the association of fish with these other behavioural patterns varies in different populations. It is also possible that negative confounding could explain the lack of association in the current study. We adjusted for other lifestyle factors such as obesity and physical activity, but cannot exclude residual negative confounding. The lack of consistency in findings between women and men in the relationship between oily fish intake and stroke suggests differential confounding.

While the existing literature suggests a potential benefit of high intake of fish in reducing risk of stroke, particularly ischaemic stroke, this may only relate to certain sorts of fish such as fish high in $n-3$ fatty acids. High fish intake that consists mainly of fish low in $n-3$ fatty acids may not have similar effects.

In summary, we found no consistent relationship between fish intake and stroke risk in men and women. Inconsistencies in the observed health effects of fish consumption may reflect different patterns, type of fish consumed and preparation methods in different populations.

\section{Acknowledgements}

We would like to thank the participants and general practitioners who took part in the study. We also thank the staff of EPIC-Norfolk and our funders.

Funding: EPIC-Norfolk is supported by research programme grant funding from Cancer Research UK and the Medical Research Council, with additional support from the Stroke Association, the British Heart Foundation, the Department of Health, the Food Standards Agency, Research into Ageing, Academy of Medical Sciences and the Wellcome Trust.

\section{Conflict of interest: None.}

Ethics approval: Norwich Local Research Ethics Committee approved the study. The corresponding address for the LREC is Clinical Governance Department, Aldwych House, 57 Bethel Street, Norwich, UK.

Contributors: K.-T.K., S.A.B., N.E.D. and N.J.W. are principal investigators in the EPIC-Norfolk population study. R.N.L. is responsible for data management, computing and data linkages. P.K.M. conducted the analysis. All co-authors contributed to the writing of this paper. K.-T.K. is the guarantor.

\section{References}

1 Burr ML, Fehily AM, Gilbert JF, Rogers S, Holliday RM, Sweetnam PM, et al. Effects of changes in fat, fish, and fibre intakes on death and myocardial reinfarction: Diet and Reinfarction Trial (DART). Lancet 1989; 2: 757-61.
2 Ness AR, Hughes J, Elwood PC, Whitley E, Smith GD, Burr ML. The long-term effect of dietary advice in men with coronary disease: follow-up of the Diet and Reinfarction trial (DART). European Journal of Clinical Nutrition 2002; 56: 512-8.

3 He K, Rimm EB, Merchant A, Rosner BA, Stampfer MJ, Willett WC, et al. Fish consumption and risk of stroke in men. Journal of the American Medical Association 2002; 288: 3130-6.

4 Yuan JM, Ross RK, Gao YT, Yu MC. Fish and shellfish consumption in relation to death from myocardial infarction among men in Shanghai, China. American Journal of Epidemiology 2001; 154: 809-16.

5 Caicoya M. Fish consumption and stroke: a community case-control study in Asturias, Spain. Neuroepidemiology 2002; 21: 107-14.

6 He K, Song Y, Daviglus ML, Liu K, Van Horn L, Dyer AR, et al. Fish consumption and incidence of stroke. A meta-analysis of cohort studies. Stroke 2004; 35: 1538-42.

7 Day N, Oakes S, Luben R, Khaw KT, Bingham S, Welch A, et al. EPIC-Norfolk: study design and characteristics of the cohort. British Journal of Cancer 1999; 80: 95-103.

8 Lohman T, Roche A, Martorell R. Anthropometric Standardization Reference Manual. Champaign, IL: Human Kinetics Books, 1991.

9 Friedewald WT, Levy RI, Fredrickson DS. Estimation of the concentration of low-density lipoprotein cholesterol in plasma, without use of the preparative ultracentrifuge. Clinical Chemistry 1972; 18: 499-502.

10 Welch AA, Luben R, Khaw KT, Bingham SA. The CAFE computer program for nutritional analysis of the EPIC-Norfolk food frequency questionnaire and identification of extreme nutrient values. Journal of Human Nutrition and Dietetics 2005; 18: 99-116.

11 Wareham NJ, Jakes RW, Rennie KL, Schuit J, Mitchell J, Hennings S, et al. Validity and repeatability of a simple index derived from the short physical activity questionnaire used in the European Prospective Investigation into Cancer and Nutrition (EPIC) study. Public Health Nutrition 2003; 6: 407-13.

12 Cox DR. Regression models and life tables. Journal of the Royal Statistical Society Series B 1972; 34: 187-220.

13 Skerrett PJ, Hennekens CH. Consumption of fish and fish oils and decreased risk of stroke. Preventive Cardiology 2003; 6: $38-41$.

14 Keli SO, Feskens EJ, Kromhout D. Fish consumption and risk of stroke. The Zutphen Study. Stroke 1994; 25: 328-32.

15 Iso H, Rexrode KM, Stampfer MJ, Manson JE, Colditz GA, Speizer FE, et al. Intake of fish and omega-3 fatty acids and risk of stroke in women. Journal of the American Medical Association 2001; 285: 304-12.

16 Morris MC, Manson JE, Rosner B, Buring JE, Willett WC, Hennekens $\mathrm{CH}$. Fish consumption and cardiovascular disease in the Physicians' Health Study: a prospective study. American Journal of Epidemiology 1995; 142: $166-75$.

17 Gillum RF, Mussolino ME, Madans JH. The relationship between fish consumption and stroke incidence. The NHANES I Epidemiologic Follow-up study (National Health and Nutrition Examination Survey). Archives of Internal Medicine 1996; 156: 537-42.

18 Mozaffarian D, Longstreth WT, Lemaitre RN, Manolio TA, Kuller LH, Burke GL, et al. Fish consumption and stroke risk in elderly individuals. The Cardiovascular Health Study. Archives of Internal Medicine 2005; 165: 200-6.

19 Welch AA, Lund E, Amiano P, Dorronsoro M, Brustad M, Kumle M, et al. Variability of fish consumption within the 10 European countries participating in the European 
Investigation into Cancer and Nutrition (EPIC) study. Public Health Nutrition 2002; 5: 1273-85.

20 Orencia AJ, Daviglus ML, Dyer AR, Shekelle RB, Stamler J. Fish consumption and stroke in men. 30-year findings of the Chicago Western Electric Study. Stroke 1996; 27: 204-9.

21 Simopoulos AP. Omega-3 fatty acids in inflammation and autoimmune diseases. Journal of the American College of Nutrition 2002; 21: 495-505.
22 Christensen JH. $n$-3 Fatty acids and the risk of sudden cardiac death. Emphasis on heart rate variability. Danish Medical Bulletin 2003; 50: 347-67.

23 GISSI-Prevenzione Investigators. Dietary supplementation with $n-3$ polyunsaturated fatty acids and vitamin $\mathrm{E}$ after myocardial infarction: results of the GISSI-Prevenzione trial. Gruppo Italiano per lo Studio della Sopravvivenza nell'Infarto miocardico. Lancet 1999; 354: 447-55. 